\title{
Predicting the statistics of wave transport through chaotic cavities by the Random Coupling Model: a review and recent progress
}

\author{
Gabriele Gradoni, Jen-Hao Yeh, Bo Xiao, Thomas M. Antonsen, \\ Steven M. Anlage, and Edward Ott \\ Institute for Research in Electronics and Applied Physics, \\ Department of Physics, \\ Department of Electrical and Computer Engineering \\ University of Maryland, \\ College Park, MD-20742, USA
}

\begin{abstract}
In this review, a model (the Random Coupling Model) that gives a statistical description of the coupling of radiation into and out of large enclosures through localized and/or distributed channels is presented. The Random Coupling Model combines both deterministic and statistical phenomena. The model makes use of wave chaos theory to extend the classical modal description of the cavity fields in the presence of boundaries that lead to chaotic ray trajectories. The model is based on a clear separation between the universal statistical behavior of the isolated chaotic system, and the deterministic coupling channel characteristics. Moreover, the ability of the random coupling model to describe interconnected cavities, aperture coupling, and the effects of short ray trajectories is discussed. A relation between the random coupling model and other formulations adopted in acoustics, optics, and statistical electromagnetics, is examined. In particular, a rigorous analogy of the random coupling model with the Statistical Energy Analysis used in acoustics is presented.
\end{abstract}

Keywords: Electromagnetic environment (EME), complex cavity, wave chaos, statistical modeling, impedance matrix, admittance matrix, short-range orbits, antenna, aperture, interconnected systems, statistical energy analysis (SEA). 


\section{Introduction}

The random coupling model (RCM) addresses the problem of statistically modeling the wave behavior of large irregular cavities connected to an external environment by one or more channels. The RCM is formulated in terms of impedance, a concept common to both the electromagnetic and acoustics communities. As such, many of the lessons learned in an electromagnetic context presented below have close analogs in the field of vibro-acoustics.

Increasingly complex scenarios in electronics and telecommunications make the detail of structures and circuitry more and more difficult to model. In addition, often in optics, electronics, and acoustics, the need for higher bitrates pushes wave sources to emit at very short wavelengths compared to the characteristics size of the excited system. In this regime, the scattering process can be very sensitive to details. A statistical approach then becomes appropriate. Specifically, one can ask what the statistics of quantities of interest are relative to a suitable random choice of the system. This is the goal of the RCM. Other statistical approaches have already been used to study reverberation chambers and wireless propagation channels. For example, a Gaussian state is assumed a priori for the complex Cartesian field upon maximization of entropy Hill (2009).

The RCM approach involves two physical prescriptions inherited from the quantum chaos theory of compound nuclei in nuclear reaction theory Wigner (1967); Mitchell et al. (2010). The first prescription is to replace the exact spectrum of the cavity with a spectrum whose statistics are obtained from those of a suitable ensemble of random matrices. The second prescription, motivated by the typical chaotic behavior of rays in complex enclosures, is to take for the mode amplitude a superposition of random plane-waves.

The applicability of Random Matrix Theory (RMT) to the solution of simple wave equations in bounded regions where the corresponding ray trajectories are chaotic was originally explored by McDonald and Kaufman (1979).

Understanding the influence of ports (antennas/sensors) and apertures on cavity field dynamics is itself interesting in practice. The chaotic regime of fields inside an irregular cavity is established by the boundary geometry driving ray propagation. From experimental observations, it is known that this results in a very high variability of the wave properties of a system. The RCM incorporates this in a formally elegant and yet physically meaningful way.

The main goal in formulating the RCM was to connect the behavior pre- 
dicted by random matrix theory (RMT) developed for the case of isolated wave chaotic systems, e. g., closed cavities, to the study of the behavior of such a system when it is connected to other systems through deterministic wave propagation channels, and to investigate the resulting input/output system characteristics. This was done in the quantum mechanical context, for example, by Brouwer (1995). The RCM was developed for resonators with spatially localized channels Zheng (2005). In 2006, studies were published for both the single channel Zheng et al. (2006a), and the multiple channel Zheng et al. (2006b) cases. Those two papers developed previous studies considering isolated chaotic systems exhibiting Hamiltonian chaos Ott (2002).

The RCM attacks this problem from the point of view of port impedance/admittance matrices, in which the universal fluctuation of fields within the cavity is joined to the specific radiation characteristics of deterministic channels, whose geometry is presumed to be known a priori.

\section{Mathematical model}

The wave chaos inside the cavity is expressed as a universal (i.e., system independent) superposition of "chaotic" modes resulting in a universal ensemble of matrices, and the presence of channels connected with the external environment results in "dressing" this superposition with the radiation impedance matrix $Z^{\text {rad }}$ of channel terminals. In the case of a cavity with a single port the cavity impedance $Z^{\text {cav }}$ can be expressed as

$$
Z^{c a v}=i \Im\left\{Z^{r a d}\right\}+\Re\left\{Z^{r a d}\right\} \xi,
$$

where $Z^{(\mathrm{rad})}$ is a port radiation impedance that will be defined later in the paper, $\xi$ is a statistically fluctuating variable corresponding to a sum over chaotic modes, and $\Im\{\cdot\}$ and $\Re\{\cdot\}$ denote imaginary and real parts. Thus, the statistics of $Z^{c a v}$ is determined by the statistics of the random matrix $\xi$ and by the nonstatistical matrix $Z^{\text {rad }}$. The sum over chaotic modes is universal in the sense that it depends only on a single parameter capturing

cavity losses and modal structure through the average quality factor Arnaut (2003) and the mean spacing between nearest neighbor eigenmodes Wigner (1967) and on no other cavity details.

Recently, the RCM has been developed for three dimensional cavities with distributed ports, e.g., complicated antennas, in the same form of (1) Antonsen et al. (2011). The presence of apertures calls for a different modeling attack, leading to the same form of the RCM for the cavity admittance 
matrix. The problem of external radiation coupling to the cavity through an aperture has been solved Gradoni et al. (2012b,a). The RCM introduces and incorporates system-specific characteristics efficiently. It will be also shown how deviations from pure wave chaos can be included in the RCM by considering short-ray orbits Ishio and Burgdörfer (1995); Baranger and Mello (1996). The RCM impedance expression (11) is preserved and the nonuniversal term is augmented by tracing those "non-chaotic" short ray orbits Hart et al. (2009).

In realistic physical scenarios one may employ different kinds of channel coupling to a closed electromagnetic environment (EME). Imagine a situation involving compartments of ships/aircraft/automobiles/trains: there could be present very localized terminals belonging to circuitry located inside bays, as well as very large windows and ports. It is thus quite useful to formulate a "hybrid" RCM involving both port terminals and electrically wide apertures, and to consider interconnected cavities.

In this paper, the general formulation of the RCM for ports and apertures is reviewed. Then, the predictions of probability distribution functions of desired input/output parameters (e.g., impedances, admittances or scattering parameters) obtained for single-mode channels by Monte Carlo computation of the RCM, and their experimental confirmation by measurements on actual systems are recalled. Finally, an application of the model to a situation of practical interest is discussed: the modeling of linear chains of interconnected chaotic cavities Gradoni et al. (2012d). Interestingly, the formula obtained for the power flowing through a chain of lossy chaotic cavities is similar to the statistical energy analysis (SEA) approach developed for the propagation of acoustic and vibrational waves.

\section{Historical remarks and methods}

Beginning in 1982 there was rapid development in the application of RMT to both quantum and classical chaotic systems. The first use of RMT was devoted to reproducing the spacing distribution of energy levels in compound nuclei. Later Rydberg levels of the hydrogen atom in a strong magnetic field, and elastomechanical eigenfrequencies of irregularly shaped quartz blocks were studied, as well as fluctuations of conduction through mesoscopic wires in a magnetic field Guhr et al. (1998); Weidenmüller and Mitchell (2009); Mitchell et al. (2010). Studies on quantum chaos of quantum transport are referenced in the reviews Beenakker (1997); Alhassid (2000). However, only 
a very small number of experiments on wave-chaotic scattering and eigenfrequencies of electromagnetic cavities existed. The first studies of irregularly shaped microwave cavities Stöckmann and Stein (1990); Doron et al. (1990); Sridhar (1991); Gräf et al. (1992) paved the way to electromagnetic wavechaotic scattering research. Microwave cavities with irregular shapes (having ray trajectories evolved by a chaotic map) provided a simple and effective physical framework for the study of wave-chaos, where not only the magnitude, but also the phase of scattering coefficients, can be directly measured from experiments. The picture of rays is analogous to that of particles bouncing in a confined system with irregular potential barriers, whose trajectories exhibit exponential divergence in phase space, i.e., two particles originating from the same position with slightly different linear momentum follow very different paths after a few bounces off the boundaries. This dynamic effect has an impact on the spectrum of complex wave systems that are chaotic in the classical limit, which can be modeled by an unpredictable (random) Hamiltonian.

The derivation of the RCM exploits both the Wigner surmise Wigner (1967) and the Berry hypothesis Berry (1977). If the dimensions of the cavity are much greater than the excitation wavelength, the semiclassical regime can be invoked. Irregular boundaries create highly disordered mode topologies. Therefore, the mode amplitude is locally modeled as an isotropic random superposition of plane waves (the Berry hypothesis). The motivation of this hypothesis is that the orbits of rays in a fully chaotic system visit all regions of phase space ergodically. In particular, if some time along a long orbit is randomly chosen, the probability density of the orbit direction is uniform in 0 to $2 \pi$. At the same time, the resonant behavior of modes is preserved by using RMT to generate mode wavenumbers (the Wigner surmise). Here, eigenvalues of a random matrix of the Gaussian Orthogonal Ensemble (GOE) with Gaussian distributed elements have been used since the time-reversal invariance is assumed to hold. The present approach is not phenomenological as the invoked ensemble when using RMT expresses the symmetry properties of the chaotic class a cavity belongs to (see So et al. (1995) for experimental work where "time reversibility" is violated by the presence of a magnetized ferrite).

This motivates the use of the Gaussian ensembles. However, in the presence of losses and interactions with the external environment, e.g., reactions in compound nuclei Mitchell et al. (2010), the universal properties of a system are intimately modified. For resonant electromagnetic systems, 
a unified model describing the behavior of open systems with losses was generated by several groups Savin et al. (2001); Fyodorov and Savin (2004); Savin and Sommers (2004); Fyodorov et al. (2005); Savin et al. (2005). Several extensions and experimental validation were published Hemmady et al. (2005b,a, 2006b,a); Zheng et al. (2006c); Yeh et al. (2010b,a, 2012b,a); Hemmady et al. (2012) related to the theory of Zheng et al. (2006a,b, c) . After that, an effort was made to incorporate imperfections arising from the interaction of ports with nearby objects and cavity walls, giving rise to short-orbits Hart et al. (2009). More recently, the model has been extended to 3D cavities with distributed ports Antonsen et al. (2011), and to account for the interaction through arbitrary apertures Gradoni et al. (2012c). Interestingly, the electrical formulation underlying the RCM is formally and physically similar to other formulations involving complex wave systems of different physical nature, e.g., acoustics. In the following, the analogy between the RCM and statistical approaches to understanding the properties of complex wave structures in applied electromagnetics, acoustics \& vibrations, and random lasers is briefly discussed.

In statistical electromagnetics (SEM), statistics and stochastic analysis are used to model complex electromagnetic systems Holland and John (1999). The aim is, besides conceiving simple and effective design tools, to shed light on the collective behavior of modes and rays when subject to irregular and heterogeneous boundaries. Cavities and resonators constitute a natural and fertile terrain for SEM to be developed and applied. A number of models have been proposed to explain and predict fluctuations inside cavities (see for example Hill (2009)). The RCM improves on those phenomenological theories by describing random fluctuations through statistical ensemble theories Schwabl and Brewer (2006). In contrast, the RCM builds on the exact model of impedance and admittance matrices as derived from Maxwell's equations.

As previously remarked, the use of RMT in physical systems dates back to 1950, when Wigner and Dirad (1950) adopted symmetry properties of statistical ensembles to model the Hamiltonian of complex nuclear systems. Besides nuclear theory, early applications of RMT can be found in acoustics and vibration systems Couchman et al. (1992); Tanner and Sondergaard (2007); Wright and Weaver (2010). The pure statistical perspective of physical systems is even older and originates from thermodynamics. Interestingly, this perspective led spontaneously to the use of random matrices for modeling transmission of vibrational energy. Specifically, a model in acoustics that is 
close to those in electromagnetics can be found in the so-called statistical energy analysis (SEA) Lyon (2003). This method is currently used as a key strategy in developing numerical tools for analysis of vibrational energy in complex mechanical systems Maksimov and Tanner (2011).

Applications of RMT can be also found in the field of optics and photonics. As an example, chaotic cavities are used to create several irregular modes that eventually lock within a narrow localization bandwidth thus creating lasing, whence the name "random laser" Cao et al. (1999); Türeci et al. (2006).

\section{Theory}

The RCM is conveniently formulated in terms of an impedance or admittance matrix. The use of network theory for solving electromagnetic problems is widely accepted in engineering and physics Felsen et al. (2007). This often leads to theoretical formulae that can be efficiently computed and compared with actual measurements. In particular, the use of open-circuit parameters such as impedances and admittances does not require any specific knowledge of the sources and detectors connected to the terminals where voltages and currents are supposed to exist. Imagine injecting an excitation current into a small antenna radiating inside an irregular cavity. The multiple reflections and scattering of rays off the metallic (lossy) walls establish a reaction to the current density flowing on the antenna surface. This creates an electromotive force that builds up the response voltage at the terminals of the antenna. In the case of a single port, the ratio of the reaction voltage over the excitation current defines the terminal impedance. This parameter has affinities with the concept of acoustic impedance, defined by the ratio of the acoustic pressure, analogous to the reaction voltage in electromagnetic systems, and the acoustic volume flow, analogous to the electrical excitation current. The acoustic impedance at a particular frequency indicates how much sound pressure is generated by a given air vibration at that frequency.

In the specific case where the antenna radiated inside a cavity, the impedance is called the cavity impedance Zheng et al. (2006a). In the presence of multiple ports, the response effect of the excitation current on each antenna

terminal can be easily separated by defining a cavity impedance matrix Zheng et al. (2006a).

$$
V_{p}=\sum_{p^{\prime}} Z_{p p^{\prime}}^{c a v}\left(k_{0}\right) I_{p^{\prime}}
$$


To find $Z_{p p^{\prime}}^{\text {cav }}$ in 3D cavities, a solution of the vector wave equation satisfying cavity boundary conditions is constructed. This is done through the usual procedure of expanding the cavity fields in a basis of electromagnetic and electrostatic eigenmodes. At this point, the prescriptions of the RCM based on wave chaos and random matrix theory are used. Firstly, the exact cavity spectrum is replaced with a spectrum of eigenvalues generated by a large random matrix of the GOE. The symmetry properties of the adopted matrix reflect the physical properties of the actual system, whence other ensembles can be invoked for generating the random eigenvalues, e.g., the Gaussian Unitary Ensemble (GUE) in case the time-reversal invariance is broken by the presence of a magnetized ferrite. Secondly, the mode amplitude (which is unknown) is replaced with a superposition of random plane-waves. Those two ingredients define what we call a "chaotic mode" of the irregular cavity.

Starting from a deterministic mode expansion, the RCM prescriptions lead to a fluctuating impedance matrix having dimension $N_{p} \times N_{p}$, where $N_{p}$ is the number of ports connected to the cavity, viz.,

$$
\underline{\underline{Z}}^{\text {cav }}=i \Im\left\{\underline{\underline{Z}}^{\mathrm{rad}}\right\}+\left[\Re\left\{\underline{\underline{Z}}^{\mathrm{rad}}\right\}\right]^{1 / 2} \cdot \underline{\underline{\xi}} \cdot\left[\Re\left\{\underline{\underline{Z}}^{\mathrm{rad}}\right\}\right]^{1 / 2},
$$

where $\underline{Z}^{\text {rad }}=\underline{\underline{R}}^{\text {rad }}+i \Im\left(\underline{Z}^{\text {rad }}\right)$ is in the simplest theory an $N_{p} \times N_{p}$ diagonal matrix whose elements are the complex radiation impedances of each port. Here, the radiation impedance provides the linear relation between voltages and currents at a port in the case in which waves are allowed to enter the enclosure through the port but not return, as if they were absorbed in the enclosure. The matrix $\underline{\underline{\xi}}$ naturally incorporates cavity homogeneous losses, and it is defined as

$$
\underline{\underline{\xi}}=-\frac{i}{\pi} \sum_{n} \frac{\underline{\Phi}_{n} \underline{\Phi}_{n}^{T}}{\mathcal{K}_{0}^{2}-\mathcal{K}_{n}^{2}+i \alpha},
$$

where $\mathcal{K}_{(\cdot)}^{2}=k_{(\cdot)}^{2} / \Delta k^{2}$, and $\alpha$ is the loss parameter defined below. In the lossless case, $\xi$ is found to be an element of the Lorentzian ensemble Brouwer and Beenakker (1997). Here, $\underline{\Phi}_{n}$ is a vector of uncorrelated, zero mean, unit width Gaussian random variables, and $k_{n}^{2}$ are the eigenvalues of a matrix selected from the GOE Mehta (1991), where the central eigenvalue is shifted to be close to $k_{0}^{2}=\omega^{2} / c^{2}$, and $\omega$ is the frequency of excitation. In case of high number of overlapping modes, e.g., occurring in overmoded reverberation chambers, the eigenvalues have uniform distribution and are distributed as per Wigner's "semicircle law" (see (Hemmady et al., 2012, 
Appendix) and Mehta (1991) for more details). Eigenvalues associated with participating modes are scaled so that the average spacing between eigenvalues near the central one is $\Delta k^{2}$, which is selected to match the mean spacing of resonances of the enclosure in the frequency range of interest. The effect of wall losses, internal homogeneous losses and the cavity dimensions (volume), are embedded into a single dimensionless loss parameter

$$
\alpha=\frac{k_{0}^{2}}{Q \Delta k^{2}},
$$

appearing in (4), which is formally identical to the inverse finesse parameter, widely used in optical resonators to quantify the granularity of resonances over a frequency band of interest Born and Wolf (1999), and where the (average) cavity quality factor is defined as

$$
Q=\omega \frac{E}{P_{d}}
$$

with $E$ the average energy stored inside, and $P_{d}$ total power dissipated throughout the cavity. The greater $\alpha$ the higher the resonance overlapping over the considered bandwidth.

It is through the matrix $\underline{\xi}$ that the propagation of waves in the enclosure from one port to another and back is modeled. Such a propagation is universally fluctuating with statistics regulated by a single parameter, $\alpha$. The RCM treats ports by means of a slowly-varying-in-frequency radiation impedance matrix. The enclosure itself is modeled by (4) that has the physical meaning of a normalized impedance, modeling the chaotic scattering taking place throughout the cavity. Rapid variations of impedance with frequency arise from this chaotic behavior. Similarly, in acoustics and vibrational systems, the acoustic impedance usually varies strongly when the frequency is changed. The form (3) makes a perfect link with well-established deterministic theories. The novelty of the RCM resides in the definition of a 'random' impedance that is dressed with deterministic features.

In practical mode-stirred chambers, it is expected that perfect mixing leads to a zero mean complex field. However, an offset in the scatter plot of the measured field is often experienced. By adopting the Poisson kernel theory, the RCM has been extended to account for short-range ray trajectories (short-orbits) between the ports. Those short orbits create system-specific properties resulting in nonuniversal frequency fluctuations, and can be acconted for with an extension of the RMT. 
The philosophy of the Poisson kernel Mello et al. (1985); Kuhl et al. (2005) is to include those scattering matrix contributions arising from parts of the system that are non-random. This same perspective has been applied to the impedance matrix $\underline{Z}$ of a microwave enclosure Hart et al. (2009). Experiments involving mode-stirred cavities and ray-chaotic cavities often rely on the variation of boundary configuration to create several statistically independent realizations of the system and to compile statistics Kostas and Boverie (1991); Schäfer et al. (2005); Köber et al. (2011). However, imperfections in the realization of perfectly diffused fields have been observed Primiani et al. (2009) numerically and experimentally. This resulted in the beginning of a serious effort to model and understanding these "unstirred" occurrences Primiani and Moglie (2010); Pirkl et al. (2011). From a wave chaotic perspective, it is noted that the channel-channel interaction introduces deterministic field components that remain fixed throughout the ensemble (such as wall proximity on scattering objects inside the enclosure) despite the design of a very efficient stirring structure Moglie and Primiani (2012). This kind of "imperfection" has been widely investigated in quantum chaos Prange (2005); Bulgakov et al. (2006). Therefore, relevant ray trajectories, which remain unchanged in many or all realizations of the ensemble, may exist and represent another system-specific feature. Hart et al. Hart et al. (2009); Yeh et al. (2010b, a) extended the RCM to take account of such ray trajectories that leave a port and soon return to it, or another port, instead of ergodically sampling the system. These ray trajectories are also called "short orbits" or "short trajectories". It is imagined that each ray leaves from a port $p$ at $\mathbf{r}_{i}$ with a certain $\mathbf{k}_{i}$ vector and it arrives at a final position $\mathbf{r}_{f}$ with a wave vector $\mathbf{k}_{f}$. It is further assumed that the ports are separated from the wall much more than a wavelength.

The impedance form of the RCM is slightly modified by the presence of short-range trajectories. In particular, it is found by a semiclassical theory of orbits Hart et al. (2009)

$$
\underline{\underline{Z}}^{\text {cav }}=i \Im\left\{\underline{\underline{Z}}^{r a d}\right\}+\underline{\underline{v}}^{\dagger} \cdot \frac{\underline{\underline{\underline{1}}}+\underline{\underline{T}}}{\underline{\underline{1}}-\underline{\underline{T}}} \cdot \underline{\underline{v}},
$$

where $\underline{\underline{T}}$ is the Bogolomny transfer operator which in the electromagnetic case is given by Bogomolny (1992)

$$
T\left(q_{i}, q_{f}, \mathbf{k}\right)=\frac{-i}{4} \sqrt{D_{\mathbf{r}_{i}, \mathbf{r}_{f}}} e^{i S\left(\mathbf{r}_{i}, \mathbf{r}_{i}, \mathbf{k}\right)-i \frac{\pi}{4}} \sqrt{\frac{\cos \theta_{f}}{\cos \theta_{i}}},
$$


where $\theta_{i}\left(\theta_{f}\right)$ is the angle between the initial (final) wave vector and the surface at the position it leaves (hits), $S\left(\mathbf{r}_{i}, \mathbf{r}_{f}, \mathbf{k}\right)$ is the classic action along the direct trajectory from $\mathbf{r}_{i}$ to $\mathbf{r}_{f}$, and $\sqrt{D_{\mathbf{r}_{i}, \mathbf{r}_{f}}}$ is the stability of the orbit from $\mathbf{r}_{i}$ to $\mathbf{r}_{f}$ (monodromy) that physically represents a geometrical factor of the trajectory taking account of the spreading of the ray tube along its path. It is also found that

$$
\underline{\underline{v}}^{\dagger} \cdot \underline{\underline{v}}=\Re\left\{\underline{\underline{Z}}^{\mathrm{rad}}\right\} .
$$

Ultimately, the correction introduced by the presence of short-orbits in the $\mathrm{RCM}$ average impedance as

$$
\left\langle\underline{\underline{Z}}^{\text {cav }}\right\rangle=i \underline{\underline{X}}^{r a d}+\left[\underline{\underline{R}}^{r a d}\right]^{1 / 2} \cdot \underline{\underline{z}} \cdot\left[\underline{\underline{R}}^{r a d}\right]^{1 / 2}
$$

where the elements of $\underline{\underline{z}}$ are expressed as

$$
z_{n, m}=\sum_{b(n, m)}-p_{b} \sqrt{D_{b}} \exp \left[-\left(i|\mathbf{k}|+k^{\prime}\right) L_{b}-i|\mathbf{k}| L_{\text {port }}-i \beta_{b} \pi\right]
$$

with $b(n, m)$ an index over all classical trajectories which leave the $n^{\text {th }}$ port, bounce $\beta_{b}$ times, and return to the $m^{\text {th }}$ port, $L_{b}$ is the length of the trajectory $b, k^{\prime}=k /(2 Q)$ is the effective attenuation parameter taking account of loss through the average quality factor $Q$, and $L_{\text {port }}$ is the port-dependent constant length between the $n^{\text {th }}$ and $m^{\text {th }}$ port. The geometrical factor $D_{b}$ is now a function of the length of each segment of the trajectory, the angle of incidence of each bounce, and the radius of curvature of each wall encountered in that trajectory. The application of this theory to reverberation chambers could pave the way to understand and use the single-frequency regime, where comparison of experimental and theoretical statistics often exhibits strong deviation from asymptotic distributions Primiani et al. (2009).

Researchers have examined short orbits in cases where the system and the ports can be treated in the semiclassical approximation Prange (2005) or considered the effect on eigenfunction correlations due to short orbits associated with nearby walls Urbina and Richter (2006). Theoretical results have been previously obtained that acknowledge the effects of short-orbits in the context of quantum graphs Kottos and Smilansky (2003). The RCM for microwave cavities has been confirmed by experiments Hart et al. (2009); Yeh et al. (2010a). Moreover, experiments in a 2D microwave cavity have extracted a measure of the microwave power that is emitted at a certain point in the cavity and returns to the same point after following all possible 


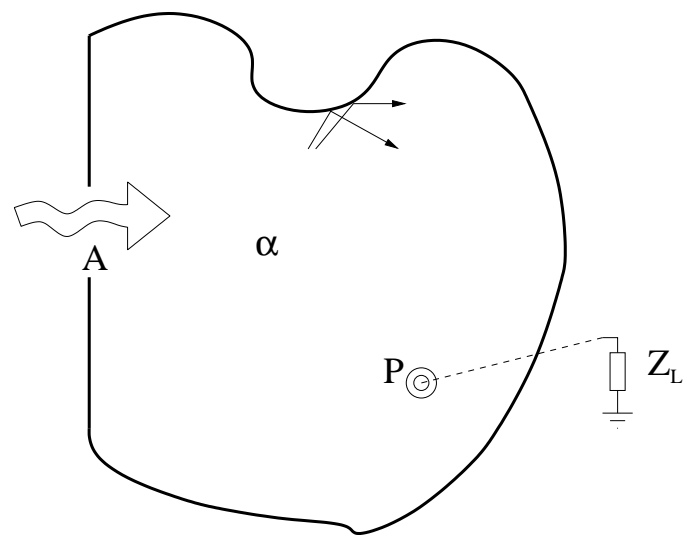

Figure 1: Wave chaotic enclosure with both one aperture and one port.

classical trajectories of a given length Stein and Stöckmann (1992). None of this prior work developed a general first-principles deterministic approach to experimentally analyze the effect of short ray trajectories. The effect of short trajectories in two-port wave-chaotic cavities has been demonstrated Yeh et al. (2010b, al) . The results of the short-orbit correction can be generalized to distributed ports and apertures by similar procedures. For apertures, it is interesting to think about the proximity of a dielectric/metallic object that is immersed in the wave-chaotic field. This physical situation has been investigated by Harrington in the case of regular cavities, where the apertureobject resonance was predicted by method of moments Harrington (1982).

\subsection{Aperture radiation of cavities}

Besides localized ports, the RCM can also include a description of apertures, a situation depicted in Fig. 1. Ongoing research is focusing on the extension to very large and irregular apertures, as well as to very small apertures, where singular fields strongly affect their (system specific) radiation Bethe (1944). The aperture excitation of an irregular enclosure is indeed a rather new theoretical problem to investigate. Interestingly, such a theory develops well on the existing literature on cavity backed apertures, and it links deterministic theories with statistical theories on reverberation chambers. The work of Harrington on cavities showed that boundary-value problems involving apertures are conveniently described in terms of the admittance matrix Harrington and Mautz (1976). Typically, radiation admittances are obtained by numerical computation of cavity eigenmodes. On the other hand, 
it is known that when the cavity is strongly perturbed by irregular objects and/or boundaries, modes change their topology and spectrum completely, e.g., mode stirred enclosures/chambers Bunting and Yu (2004), and the fields become amenable to statistical description Hill (2009). Also in the presence of apertures, thanks to the RCM perspective of separating system-specific from universal characteristics, those two apparently distinct approaches can be unified through wave chaos theory. Similar to a port, the aperture geometry is fully included in the model through the radiation admittance matrix. The procedure to be followed is practically the same that has been used to arrive at Eq. (3). A detailed mathematical derivation is described in Gradoni et al. (2012c), and is briefly recapitulated below.

The aperture has been treated as an opening in a zero thickness metallic plane, and the analysis started by expanding the aperture (tangential) field distribution in a basis of modes

$$
\mathbf{E}_{t}=\sum_{s} V_{s} \mathbf{e}_{s}\left(\mathbf{x}_{\perp}\right)
$$

where $V_{s}$ are aperture voltages representing the electric mode amplitudes, $\mathbf{x}_{\perp}$ is the spatial coordinate within the aperture area, $\mathbf{e}_{s}$ are the aperture modes and have only transverse fields, normalized such that $\int_{\text {aperture }} d x_{\perp}^{2}\left|\mathbf{e}_{s}\right|^{2}=1$. Solving Maxwell's equations with Dirichlet boundary conditions $\mathbf{E}_{t}=0$, yields an expression for transverse magnetic fields in terms of the same basis of modes

$$
\mathbf{H}_{t}=\sum_{s} I_{s} \hat{n} \times \mathbf{e}_{s}\left(\mathbf{x}_{\perp}\right)
$$

where $\hat{n}$ is the outward normal to the cavity, which has been taken to be in the $z$-direction. The linear relation between magnetic field mode amplitude $I_{s}$, and electric field mode amplitude $V_{s}$, is expressed in terms of an admittance

$$
I_{s}=\sum_{s^{\prime}} Y_{s s^{\prime}}^{(\cdot)}\left(k_{0}\right) V_{s^{\prime}}
$$

Here $Y_{s s^{\prime}}^{(\cdot)}=Y_{s s^{\prime}}^{(r a d)}$ or $Y_{s s^{\prime}}^{(\cdot)}=Y_{s s^{\prime}}^{(\text {cav })}$ depending on whether the aperture radiates into free space or into a cavity. In particular, in presence of the cavity, the boundary-value problem is solved by expanding the cavity fields in modes, and then by applying the RCM prescriptions for the properties of these modes. In this case, the RCM formulation leads to a specific relation 


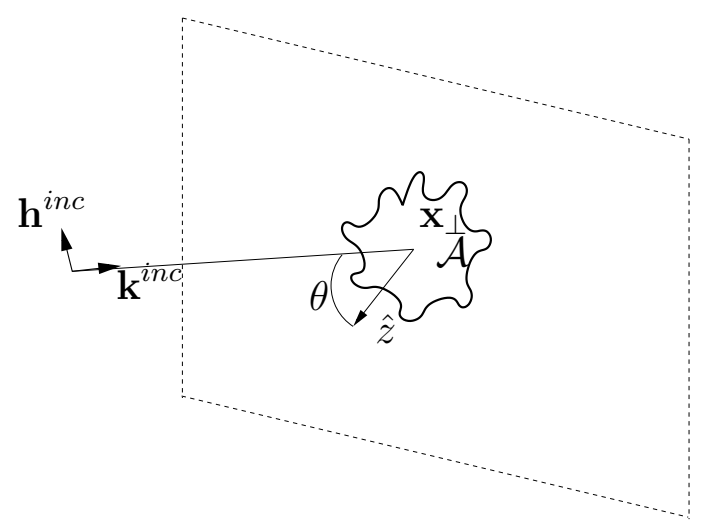

Figure 2: Geometry of an aperture of arbitrary shaper illuminated by an external plane-wave.

between free-space admittance $Y_{s s^{\prime}}^{(r a d)}$ and cavity admittance $Y_{s s^{\prime}}^{(c a v)}$, viz.,

$$
\underline{\underline{Y}}^{c a v}=i \Im\left\{\underline{\underline{Y}}^{\text {rad }}\right\}+\left[\Re\left\{\underline{\underline{Y}}^{r a d}\right\}\right]^{1 / 2} \cdot \underline{\underline{\xi}} \cdot\left[\Re\left\{\underline{\underline{Y}}^{\text {rad }}\right\}\right]^{1 / 2}
$$

where the matrix $\underline{\xi}$ is the same as defined in (44), and the elements of $\underline{\underline{Y}}^{\mathrm{rad}}$ are defined by Gradoni et al. (2012c).

\subsection{Coupling with external radiation}

In practical electromagnetic systems, an aperture can be fed by a waveguide with the same transverse sectional shape, or it can be exposed to external radiation. In both cases the tangential field expansions (12) and (13) can be preserved. It is thus instructive to analyze the typical scenario depicted in Fig. 2 of an oblique plane-wave with wave vector $\mathbf{k}^{i n c}$ and polarization of magnetic field $\mathbf{h}^{i n c}$ (perpendicular to $\mathbf{k}^{i n c}$ ) exciting the aperture. In Harrington and Mautz (1976), this analysis has been performed by continuity of tangential magnetic field at the aperture plane, that leads to the equivalent network equation by projecting the two magnetic field expressions on the aperture basis, and equating the amplitudes Gradoni et al. (2012b, a,, $\mathrm{c}$ )

$$
\left(\underline{\underline{Y}}^{c a v}+\underline{\underline{Y}}^{r a d}\right) \cdot \underline{V}=2 \underline{I}^{i n c},
$$

where $\underline{V}$ is a vector containing the voltages $V_{s}, I_{s}^{i n c}=-\hat{n} \cdot \tilde{\mathbf{e}}_{s}\left(-\mathbf{k}_{\perp}^{i n c}\right) \times \mathbf{h}^{i n c}$, and $\tilde{\mathbf{e}}$ is the Fourier transform of the aperture basis mode for the electric field. 


\subsection{Hybrid formulation: ports and apertures}

The use and verification of the RCM for apertures through quadratic (power) measurements requires the presence of antennas/ports inside the cavity. Therefore, it becomes natural to formulate a hybrid RCM including both the aperture admittance matrix and the port impedance matrix.

The RCM has been extended to account for the joint presence of electrically wide openings of arbitrary shape and localized ports. A schematic framework of such a situation is reported in Fig. 1. In that case, an input column vector $\phi$ has been constructed that consists of the aperture voltages and terminal currents, and an output vector $\psi$ consisting of the aperture currents and terminal voltages

$$
\underline{\phi}=\left[\begin{array}{l}
\underline{V}_{A} \\
\underline{I}_{P}
\end{array}\right]
$$

and

$$
\underline{\psi}=\left[\begin{array}{l}
\underline{I}_{A} \\
\underline{V}_{P}
\end{array}\right]
$$

where $\underline{V}_{A, P}$ are the aperture and port voltages, and $\underline{I}_{A, P}$ are the aperture and port currents. These are then related by a hybrid matrix $\underline{\underline{H}}, \underline{\underline{\psi}}=\underline{\underline{H}} \cdot \underline{\phi}$, where

$$
\underline{\underline{H}}=i \Im(\underline{\underline{U}})+[\Re(\underline{\underline{U}})]^{1 / 2} \cdot \underline{\underline{\xi}} \cdot[\Re(\underline{\underline{U}})]^{1 / 2} .
$$

Here the matrices $\underline{\underline{U}}$ and $\underline{\underline{V}}$ are block diagonal, viz.,

$$
\underline{\underline{U}}=\left[\begin{array}{cc}
\underline{\underline{Y}}^{r a d} & 0 \\
0 & \underline{\underline{Z}}^{\text {rad }}
\end{array}\right]
$$

and

$$
\underline{\underline{V}}=\Re(\underline{\underline{U}}) .
$$

The dimension of $\underline{\underline{U}}$ and $\underline{\underline{V}}$ is $\left(N_{s}+N_{p}\right) \times\left(N_{s}+N_{p}\right)$, where $N_{p}$ is the number of port currents and $\bar{N}_{s}$ is the number of aperture voltages. The linear problem at hand is thus formulated in terms of matrices with relatively small dimensions, typically given by the number of ports and aperture modes used to access the electromagnetic structure. Strictly speaking, the RCM formulation has facilitated a model order reduction Felsen et al. (2007) if compared to full-wave or other statistical models of cavity fields. Usually the superposition of plane-waves or modes requires the computation of the field at each 
point of the structure, thus producing very large matrices. In the different perspective of looking at channels, the available number of degrees of freedom (typically constituted by the number of excited modes of the cavity) is reduced to the number of port profiles/aperture basis functions.

\section{Random coupling model in action: numerical simulations}

Ensembles of cavities have been generated by the application of the Monte Carlo method described in Zheng et al. (2006a, b) ; Hemmady et al. (2012). Once the system-specific properties of channels are predicted or measured separately under an $\alpha \rightarrow \infty$ (free-space) condition, Monte Carlo simulations of (4) allow for generating an ensemble of cavity impedances of the form (3) or, identically, of cavity admittances of the form (15). A proper number of realizations must be chosen to create adequate statistics close to the asymptotic behavior, which is Lorentzian in the lossless regime $(\alpha=0)$, and Gaussian in the high-loss regime $(\alpha \gg 1)$, see Fig. (3). First of all, the universally fluctuating impedances (4) is recast as

$$
\underline{\underline{\xi}}=\frac{i}{\pi} \underline{\underline{\Phi}} \cdot \frac{\underline{\underline{1}}}{\underline{\underline{\lambda}}-i \alpha \underline{\underline{1}}} \cdot \underline{\underline{\Phi}}^{T} .
$$

The matrix $\underline{\underline{\Phi}}$ is a $M \times N$ coupling matrix with each element $\Phi_{i j}$ representing the coupling between the $i^{\text {th }}$ port profile/aperture mode $(1 \leq i \leq M)$ and the $j^{\text {th }}$ chaotic mode of the cavity $(1 \leq j \leq N)$. Each $\Phi_{i j}$ is an independent Gaussian-distributed random variable of zero mean and unit variance. The matrix $\underline{\underline{\Phi}}^{T}$ is the transpose of $\underline{\underline{\Phi}}$, and $\underline{\underline{1}}$ is a $N \times N$ identity matrix. The matrix $\stackrel{\bar{\lambda}}{=}$ is an $N \times N$ diagonal matrix with a set of $\mathrm{N}$ random eigenvalues based on the GOE nearest-neighbor (Wigner) spacing distribution Wigner (1967). By repeating this procedure many times in order to give a sufficiently large ensemble of $\underline{Z}$, the statistical description of the cavity is thus generated. Conversely, starting from cavity measurements of the impedance, the universal fluctuation of the single port impedance can be calculated by normalization of experimental results, to compare to RMT predictions of the universal behavior Hemmady et al. (2005b,a). Fig. 3 reports the distribution of the real (a) and the imaginary (b) part of the normalized impedance parametrized as a function of the loss factor $\alpha$. The loss parameter of the cavity at hand is predicted from the Weyl law on the basis of the volume of the cavity, its frequency of excitation $(\omega=k c)$, and the electromagnetic 


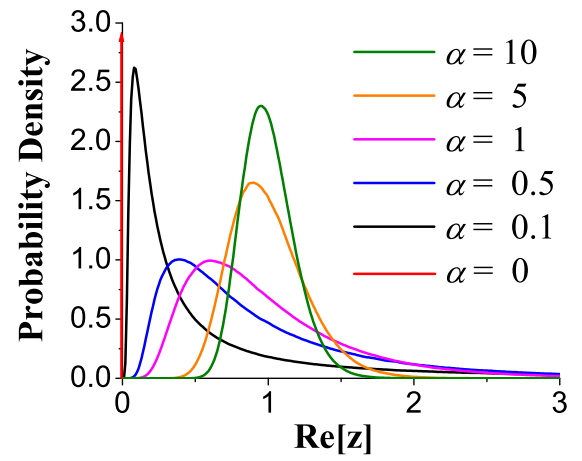

(a)

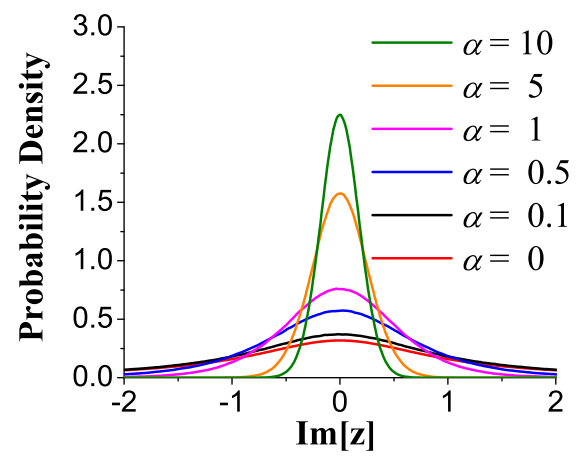

(b)

Figure 3: Random matrix theory prediction for the real (a) and the imaginary (b) part of the normalized impedance $z=\frac{Z^{c a v}-i \Im\left\{Z^{r a d}\right\}}{\Re\left\{Z^{r a d}\right\}}$ for various values of $\alpha$.

losses. For a 3D enclosure it is found

$$
\alpha=\frac{k^{3} V}{2 \pi^{2} Q},
$$

where $V$ is the cavity volume, and $Q$ the average resonator quality factor.

The effect of increasing the loss parameter results in reducing the magnitude of fluctuation of impedance elements. It is to be noticed that for $\alpha=\infty$ we retrieve the case where ports/apertures radiate in free-space. In the limit of infinitesimal losses the impedance fluctuation become very strong: this is a situation that can occur in superconducting cavities Alt et al. (1998), in low-loss optical systems, e.g., lasers Türeci et al. (2006), in microwave cavities operated in the non-Ericsson (weak overlapping) regime Dietz et al. (2008, 2009), and in reverberation chambers/enclosures operated in the undermoded regime Arnaut (2001); Warne et al. (2003); Orjubin et al. (2006); Arnaut and Gradoni (2011).

In the limit of high losses, the diagonal (off-diagonal) elements of $\underline{\underline{\xi}}$ become unit (zero) mean Gaussian random variables.

Figs. 4 (red curves) reports a few experimental results for the frequency dependence of the real (a) and imaginary (b) single-port impedance measured at the coaxial port feeding a "bowtie" ray-chaotic cavity. A high frequency 


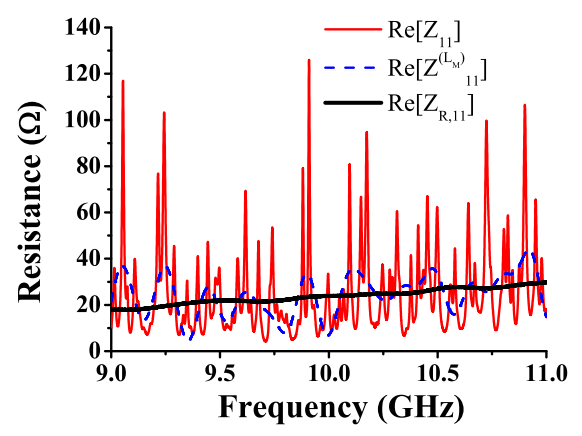

(a)

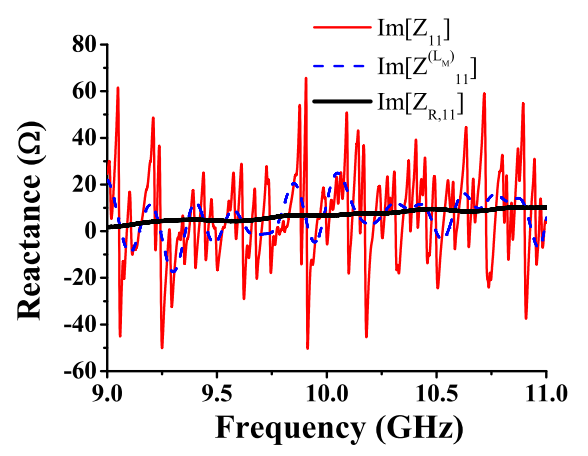

(b)

Figure 4: Resistance (a) and reactance (b) of the measured port (input) impedance in a "bowtie" cavity. The solid red curves are measured data. The dashed blue curves are created by combining the short orbit effect and the radiation impedance (thick black curves).

variability due to fluctuations that ride on top of several systematic trends can be noticed. Rapid fluctuations come from longer orbits. The first systematic trend is shown as a black solid line and represents the measured radiation impedance of the port. The second systematic trend is shown as the thin dashed curves which are semi-numerical results that contain the radiation impedance (measured) and the effect of short (less than $200 \mathrm{~cm}$ ) orbits. This should be compared to the characteristic dimension of this billiard, which is $\mathcal{L}=\sqrt{A} \approx 34 \mathrm{~cm}$. Note that the short-orbit curve follows the major trend in the data, but does not describe the rapidly varying part of the impedance. By removing all systematic effects up to an equivalent short orbit length of $\mathcal{L}_{M}=200 \mathrm{~cm}$ one can obtain a good correspondence between the theoretical and experimental normalized impedance Yeh et al. (2010a).

The question now arises: how far should the short-orbit calculation be carried? At short times only a few of the shortest orbits will contribute, whereas the number of orbits contributing to the impedance will grow exponentially as the time increases. The relevant time scale for terminating the short-orbit calculation is the Ehrenfest time. The Ehrenfest time is a time scale associated with the classical-to-quantum crossover Aleiner and Larkin (1996), and it is the time when wave packets start to deviate from the motion of deterministic classical ray trajectories to fully-developed wave chaos 


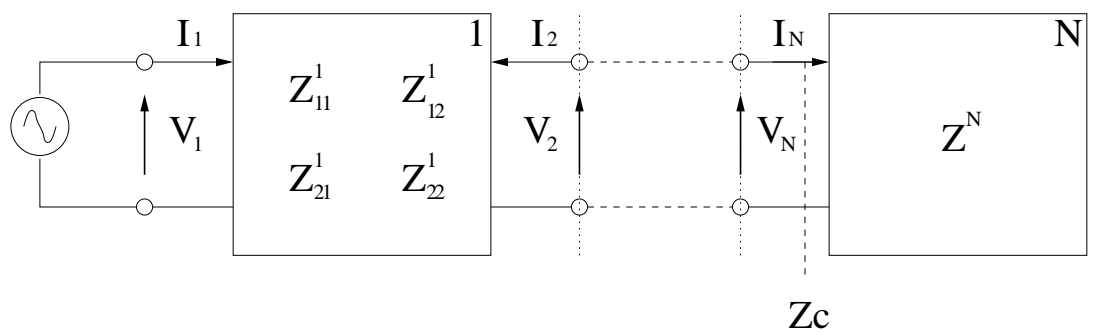

Figure 5: Network model of a chain of coupled cavities: the first cavity is excited by a localized source (port 1), and the coupled field at the last cavity is picked up by a localized detector (port $N$ ). The cavity-to-cavity coupling can take place through either single-mode channels (transmission lines) or multimode channels (distributed ports or apertures).

Schomerus and Jacquod (2005). Therefore, it is meaningless to compute the orbits whose lengths correspond to time scales much longer than the Ehrenfest time. For a microwave cavity, the Ehrenfest time can be calculated as

$$
t_{E}=\frac{1}{h} \ln \left(\frac{\mathcal{L}}{\lambda}\right)
$$

where $h$ is the largest Lyapunov exponent of the classical ray dynamics in the cavity, $\mathcal{L}$ is the characteristic dimension of the cavity, and $\lambda$ is the wavelength of the probing waves. The Ehrenfest times of the bowtie cavity and the cutcircle cavity have been estimated. The Lyapunov exponent for the bowtie billiard is $h=0.7737 \mathrm{~ns}^{-1}$, so the Ehrenfest time at $f=10 \mathrm{GHz}$ is 3.2 ns which corresponds to a length of $94 \mathrm{~cm}$ in free space. For the cut-circle billiard we found $h=0.6411 \mathrm{~ns}^{-1}$, so the Ehrenfest time at $f=10 \mathrm{GHz}$ is 3 ns which corresponds to a length of $92 \mathrm{~cm}$ in free space.

\section{Interconnection of cavities}

The RCM has been pushed further ahead to address the interconnection of complicated systems. Hence, the scenario of a linear chain of cavities has been investigated Gradoni et al. (2012d).

The analyzed physical framework consists of $N$ interconnected two-port cavities, as shown in Fig. 5. A fundamental quantity of interest for this analysis is the input impedance of the chain. This can be found in an iterated manner starting at the last cavity, where $Z_{i n}^{(N)}=Z_{11}^{(N)}$, to the first cavity, to 
find $Z_{\text {in }}^{(1)}$

$$
Z_{i n}^{(n)}=Z_{11}^{(n)}-\frac{\left(Z_{12}^{(n)}\right)^{2}}{\left(Z_{22}^{(n)}+Z_{i n}^{(n+1)}\right)}
$$

The concept of trans-impedance has been adopted as a baseline to analyze and quantify the amount of signal coupled between two or more arbitrary elements (subsystems) of the chain. In particular, for the $n$-th cavity, the voltage at the first port of the $n+1$-st cavity $V_{1}^{(n+1)}$ is related to the current exciting the first port of the $n$-th cavity $I_{1}^{(n)}$ as

$$
Z_{T}^{(n)}=\frac{V_{1}^{(n+1)}}{I_{1}^{(n)}}=\frac{Z_{i n}^{(n+1)} Z_{21}^{(n)}}{Z_{22}^{(n)}+Z_{i n}^{(n+1)}}
$$

Consequently, the ratio of power entering the $N$-th cavity to that entering the first cavity can be expressed as a product, viz.,

$$
R_{N} \equiv \frac{P_{i n}^{(N)}}{P_{i n}^{(1)}},
$$

where each impedance involved in (26) is expressed by the RCM.

The case of statistically identical cavities having moderate/high losses $\alpha_{1}=\alpha_{2}=\ldots=\alpha_{N}=\alpha>1$, deserves special attention. Correspondingly, the weak fluctuation approximation can be employed leading to a simplified expression of (26). More precisely, in the high-loss regime, $Z_{i n}^{(n)} \approx Z_{11}^{(n)}$, and fluctuating impedances can be tackled with first-order perturbation theory. This procedure results in a simplification for the denominator of (26) , and leads to an expression for the coupled power ratio $R_{N}$ that factorizes

$$
R_{N}(\alpha) \approx \prod_{n=1}^{N-1} x^{(n)} \frac{t^{(n, n+1)}}{2 \pi \alpha},
$$

where $x^{(n)}$ are exponentially distributed random variabes

$$
f_{X}(x)=\exp (-x)
$$

The transmission factors in (28) are given by

$$
t^{(n, n+1)}=\frac{R_{22, \text { rad }}^{(n)} R_{11, \text { rad }}^{(n+1)}}{\left|Z_{22, \text { rad }}^{(n)}+Z_{11, \text { rad }}^{(n+1)}\right|^{2}},
$$




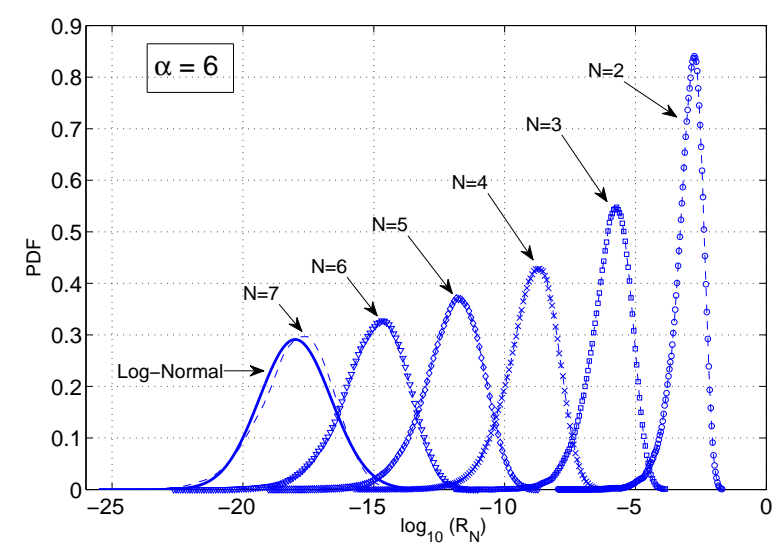

Figure 6: Probability density function of power ratios $R_{N}(\alpha=6) \equiv \frac{P_{i n}^{(N)}}{P_{i n}^{(1)}}$ for chains of up to seven cavities: the high-loss regime is assumed for all the (statistically identical) cavities in the chain.

modeling the coupling between the $n$-th and $n+1$-st cavity, and the loss factor is given by (23) $)$ In Gradoni et al. (2012d), the Monte Carlo method described in section 5 has been used to generate ensembles of impedance matrices and compute the coupling in chains of cavities. Basically, the impedance matrix for each single cavity has been generated, and used in the theoretical expressions (3) and (22). Then, the power ratio has been evaluated for sequences of cavity chains of different length. For these studies, the cavities were assumed to be statistically identical. The PDFs of the logarithm of the power ratio for the case $\alpha=6$ is shown in Fig. 6. The high loss case (Fig. 6) is well approximated by the analytic formulas (28), (29) and (30). The distribution approaches log-normal as the number of cavities becomes large. This is evident from the comparison of the 7-cavity chain with a best-fit log-normal distribution. This behavior is expected since according to (28) and (29) the power ratio becomes a product of $N$ independent and identically distributed random variables, and thus, as $N$ becomes large the logarithm of the power ratio will be normally distributed. Anderson localization in the lossless case has been observed with respect to the number of chain elements by adopting a cascaded RCM in a chaotic dynamics perspective Gradoni et al. (2012d). 


\subsection{Connection with acoustics and vibrations}

It turns out that the flow of electromagnetic energy can be expressed in a similar way to that used to model the flow of vibrational wave energy. The global system is divided into complex subsystems interconnected with each other through simple deterministic structures. In particular, Lyon (2003) applied a "thermodynamical" approach to arrive at a statistical model of energy exchange between two parts (subsystems) of the system, namely statistical energy analysis (SEA). Specifically, by using energy equipartition arguments, the average power passing from subsystem $i$ to subsystem $j\left(P_{i j}\right)$ can be expressed as (Tanner and Sondergaard, 2007, Eq. (92))

$$
P_{i j}=\omega \bar{d}_{i} \eta_{i j}\left(\frac{E_{i}}{\bar{d}_{i}}-\frac{E_{j}}{\bar{d}_{j}}\right)
$$

where $\omega$ is the mean frequency of the source, $\eta_{i j}$ is the coupling loss factor, $\bar{d}_{(\cdot)}$ is the mean density of eigenfrequencies (modes) of the uncoupled subsystems, and $E_{(\cdot)}$ is the energy stored in each subsystem. In a similar way, by recalling the average power ratio (28), it is worth noticing that the difference between the power entering two arbitrary elements of the chain can be written in the very general form

$$
P_{i j}=\frac{P_{i} T^{(i, j)}}{2 \pi \alpha^{(i)}}-\frac{P_{j} T^{(i, j)}}{2 \pi \alpha^{(j)}},
$$

valid for an arbitrary chain section of two elements, In (32), $P_{i}$ is the input power entering cavity $i$, and $P_{j}$ is the input power entering cavity $j$. In equilibrium, the net power exchange between the to subsystems is established by the power dissipated in each cavity, and by the fraction of power leaking through the small (deterministic) channel. The scheme of the physical framework analyzed to derive (32) is reported in Fig. 7. By using (5) the loss factor $\alpha^{(\cdot)}$, and by assuming

$$
\frac{P_{i}}{P_{d}^{(i)}} \approx \frac{P_{j}}{P_{d}^{(j)}}=L,
$$

where $L$ is a dissipation factor of the subsystem since $P_{d}^{(\cdot)}$ is the dissipated power within each cavity, it is found that

$$
P_{i j} \approx \omega \frac{L}{2 \pi} T^{(i j)}\left(\frac{E_{i}}{\bar{d}_{i}}-\frac{E_{j}}{\bar{d}_{j}}\right),
$$




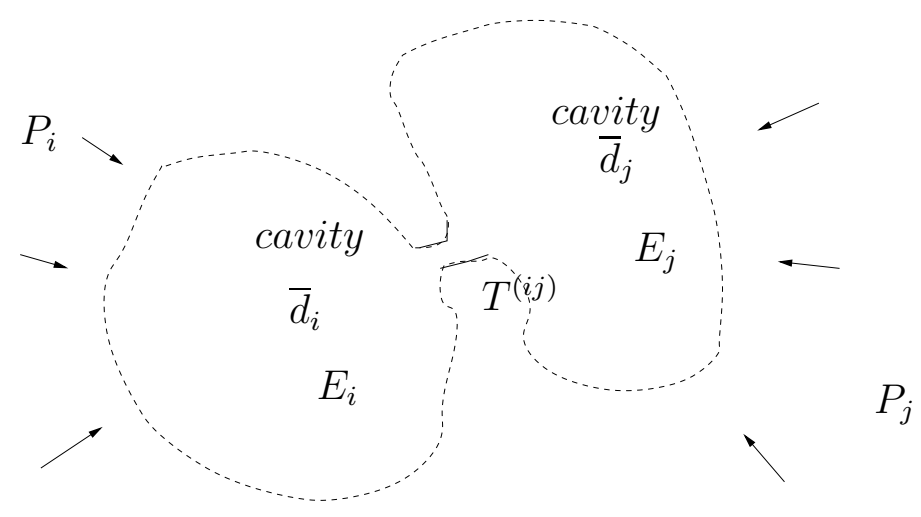

Figure 7: Arbitrary section of a chain: two cavities are excited by different input powers and they are weakly coupled. This simplified framework has been used to calculate the net power exchanged by the two cavities. A similar attack is adopted by the statistical energy analysis (SEA) approach in acoustics.

where a coupling loss factor $\eta_{i j}=L T^{(i j)} / 2 \pi$ is recognized, and

$$
\bar{d}_{(\cdot)}=\frac{k^{2}}{\Delta k_{(\cdot)}^{2}},
$$

is the mean (mode) density of each subsystem. The assumption in (33) is related to the equipartition of energy. This results in (34) being formally similar to (31) even in the case of autonomous (sub)systems coupled by an electrically short transmission line. Interestingly, in deriving the terms of (34), a weak coupling argument has been used Gradoni et al. (2012d) that is identical to the one typically used in SEA Langley (1990). Methods based on SEA have been recently integrated with the finite element method (FEM) to tackle very complex vibro-acoustic scenarios Tanner et al. (2010).

\section{Conclusion and future perspective}

The general "dressed" impedance obtained in formulating the RCM for ports and terminals radiating inside complex electromagnetic cavities has been described. This statistical model accounts for short orbit deviations from the full chaotic mixing assumption. Numerical and experimental results validate the "dressing" of a universal fluctuating impedance matrix (dependent on a single loss parameter) with radiation impedance/admittance ma- 
trices (modeling radiation of ports/apertures). A generalization of the RCM to the scenario of interconnected cavities for weak coupling conditions is presented, and the distribution of the power flowing through such a chain discussed for an increasing number of cavities. The analogy with Statistical Energy Analysis used in acoustics is pointed out by formulating a new model for the power exchanged by two wave chaotic cavities excited by different input powers.

\section{Acknowledgement}

Work supported by the Air Force Office of Scientific Research grant FA95501010106 and the Office of Naval Research grant N000140911190.

\section{References}

Aleiner, I. L., Larkin, A. I., Nov 1996. Divergence of classical trajectories and weak localization. Phys. Rev. B 54, 14423-14444.

Alhassid, Y., Oct 2000. The statistical theory of quantum dots. Rev. Mod. Phys. 72, 895-968.

URL http://link.aps.org/doi/10.1103/RevModPhys.72.895

Alt, H., Bäcker, A., Dembowski, C., Gräf, H.-D., Hofferbert, R., Rehfeld, H., Richter, A., Aug 1998. Mode fluctuation distribution for spectra of superconducting microwave billiards. Phys. Rev. E 58, 1737-1742.

URL http://link .aps .org/doi/10.1103/PhysRevE.58.1737

Antonsen, T. M., Gradoni, G., Anlage, S., Ott, E., August 2011. Statistical characterization of complex enclosures with distributed ports. In: Proceedings of the IEEE International Symposium on EMC. Long Beach, CA (USA).

Arnaut, L., nov 2001. Operation of electromagnetic reverberation chambers with wave diffractors at relatively low frequencies. Electromagnetic Compatibility, IEEE Transactions on 43 (4), $637-653$.

Arnaut, L., feb 2003. Statistics of the quality factor of a rectangular reverberation chamber. Electromagnetic Compatibility, IEEE Transactions on $45(1), 61-76$. 
Arnaut, L., Gradoni, G., aug. 2011. On distributions of fields and power in undermoded mode-stirred reverberation chambers. In: General Assembly and Scientific Symposium, 2011 XXXth URSI. pp. 1 -4.

Baranger, H. U., Mello, P. A., 1996. Short paths and information theory in quantum chaotic scattering: transport through quantum dots. Europhys. Lett. 33, 465.

Beenakker, C. W. J., 1997. Random-matrix theory of quantum transport. Rev. Mod. Phys. 69, 731.

Berry, M. V., 1977. Regular and irregular semiclassical wavefunctions. J. Phys. A 10, 2083.

Bethe, H. A., 1944. Theory of diffraction by small holes. Phys. Rev. 66 (7), $163-182$.

Bogomolny, E. B., 1992. Semiclassical quantization of multidimensional systems. Nonlinearity 5, 805.

Born, M., Wolf, E., 1999. Principles of Optics: Electromagnetic Theory of Propagation, Interference and Diffraction of Light. Cambridge University Press.

URL http://books .google.com/books?id=oV80AAAAIAAJ

Brouwer, P. W., 1995. Generalized circular ensemble of scattering matrices for a chaotic cavity with nonideal leads. Phys. Rev. B 51, 16878.

Brouwer, P. W., Beenakker, C. W. J., 1997. Voltage-probe and imaginarypotential models for dephasing in a chaotic quantum dot. Phys. Rev. B 55, 4695.

Bulgakov, E. N., Gopar, V. A., Mello, P. A., Rotter, I., 2006. Statistical study of the conductance and shot noise in open quantum-chaotic cavities: Contribution from whispering gallery modes. Phys. Rev. B 73, 155302.

Bunting, C., Yu, S.-P., may 2004. Field penetration in a rectangular box using numerical techniques: an effort to obtain statistical shielding effectiveness. Electromagnetic Compatibility, IEEE Transactions on 46 (2), 160 - 168. 
Cao, H., Zhao, Y. G., Ho, S. T., Seelig, E. W., Wang, Q. H., Chang, R. P. H., Mar 1999. Random laser action in semiconductor powder. Phys. Rev. Lett. 82, 2278-2281.

Couchman, L., Ott, E., Antonsen, T. M., Nov 1992. Quantum chaos in systems with ray splitting. Phys. Rev. A 46, 6193-6210.

Dietz, B., Friedrich, T., Harney, H. L., Miski-Oglu, M., Richter, A., Schäfer, F., Verbaarschot, J., Weidenmüller, H. A., 2009. Induced violation of timereversal invariance in the regime of weakly overlapping resonances. Phys. Rev. Lett. 103, 064101.

Dietz, B., Friedrich, T., Harney, H. L., Miski-Oglu, M., Richter, A., Schäfer, F., Weidenmüller, H. A., 2008. Chaotic scattering in the regime of weakly overlapping resonances. Phys. Rev. E 78, 055204.

Doron, E., Smilansky, U., Frenkel, A., 1990. Experimental demonstration of chaotic scattering of microwaves. Phys. Rev. Lett. 65, 3072.

Felsen, L. B., Mongiardo, M., Russer, P., 2007. Electromagnetic Field Computation by Network Methods. Springer.

Fyodorov, Y. V., Savin, D. V., 2004. Statistics of impedance, local density of states, and reflection in quantum chaotic systems with absorption. JETP Lett. 80, 725 .

Fyodorov, Y. V., Savin, D. V., Sommers, H.-J., 2005. Scattering, reflection and impedance of waves in chaotic and disordered systems with absorption. J. Phys. A: Math. Gen. 38, 10731.

Gradoni, G., Antonsen, T., Anlage, S., Ott, E., sept. 2012a. Coupling of external radiation to circuitry inside complex em environments. In: Electromagnetics in Advanced Applications (ICEAA), 2012 International Conference on. pp. $1233-1234$.

Gradoni, G., Antonsen, T., Anlage, S., Ott, E., sept. 2012b. External radiation of complex cavities described by the random coupling model. In: Electromagnetics in Advanced Applications (ICEAA), 2012 International Conference on. pp. $357-358$. 
Gradoni, G., Antonsen, T. M., Anlage, S., Ott, E., September 2012c. Theoretical analysis of apertures radiating inside wave chaotic cavities. In: Proceedings of the EMC Europe. Rome (Italy).

Gradoni, G., Antonsen, T. M., Ott, E., Oct 2012d. Impedance and power fluctuations in linear chains of coupled wave chaotic cavities. Phys. Rev. E 86, 046204. URL http://link.aps.org/doi/10.1103/PhysRevE.86.046204

Gräf, H.-D., Harney, H. L., Lengeler, H., Lewenkopf, C. H., Rangacharyulu, C., Richter, A., Schardt, P., Weidenmüller, H. A., Aug 1992. Distribution of eigenmodes in a superconducting stadium billiard with chaotic dynamics. Phys. Rev. Lett. 69, 1296-1299. URL http://link.aps.org/doi/10.1103/PhysRevLett.69.1296

Guhr, T., Müller-Groeling, A., Weidenmüller, H. A., 1998. Random-matrix theories in quantum physics: common concepts. Phys. Rep. 299, 189.

Harrington, R., mar 1982. Resonant behavior of a small aperture backed by a conducting body. Antennas and Propagation, IEEE Transactions on $30(2), 205-212$.

Harrington, R. F., Mautz, J. R., 1976. A generalized network formulation for aperture problems. IEEE Transactions on Antennas and Propagation $24(6), 870-873$.

Hart, J. A., Antonsen, T. M., Ott, E., 2009. Effect of short ray trajectories on the scattering statistics of wave chaotic systems. Phys. Rev. E 80, 041109.

Hemmady, S., Antonsen, T. M., Ott, E., Anlage, S. M., 2012. Statistical prediction and measurement of induced voltages on components within complicated enclosures: A wave-chaotic approach. IEEE Trans. Electromag. Compat. 99, 1.

Hemmady, S., Zheng, X., Antonsen, T. M., Ott, E., Anlage, S. M., 2005a. Universal statistics of the scattering coefficient of chaotic microwave cavities. Phys. Rev. E 71, 056215.

Hemmady, S., Zheng, X., Antonsen, T. M., Ott, E., Anlage, S. M., 2006a. Aspects of the scattering and impedance properties of chaotic microwave cavities. Acta Physica Polonica A 109, 65. 
Hemmady, S., Zheng, X., Antonsen, T. M., Ott, E., Anlage, S. M., $2006 \mathrm{~b}$. Universal properties of two-port scattering, impedance, and admittance matrices of wave-chaotic systems. Phys. Rev. E 74, 036213.

Hemmady, S., Zheng, X., Ott, E., Antonsen, T. M., Anlage, S. M., 2005 b. Universal impedance fluctuations in wave chaotic systems. Phys. Rev. Lett. 94, 014102 .

Hill, D., 2009. Electromagnetic Fields in Cavities: Deterministic and Statistical Theories. IEEE Press Series on Electromagnetic Wave Theory. Wiley. URL http://books.google.com/books?id=6BhWMXBf3JYC

Holland, R., John, R. S., 1999. Statistical Electromagnetics. Taylor and Francis.

Ishio, H., Burgdörfer, J., 1995. Quantum conductance fluctuations and classical short-path dynamics. Phys. Rev. B 51, 2013.

Köber, B., Kuhl, U., Stöckmann, H.-J., Goussev, A., Richter, K., Jan 2011. Fidelity decay for local perturbations: Microwave evidence for oscillating decay exponents. Phys. Rev. E 83, 016214.

Kostas, J. G., Boverie, B., 1991. Statistical model for a mode-stirred chamber. IEEE Trans. EMC 33, 366.

Kottos, T., Smilansky, U., 2003. Quantum graphs: a simple model for chaotic scattering. J. Phys. A: Math. Gen. 36, 3501.

Kuhl, U., Martínez-Mares, M., Méndez-Sánchez, R. A., Stöckmann, H. J., 2005. Direct processes in chaotic microwave cavities in the presence of absorption. Phys. Rev. Lett. 94, 144101.

Langley, R., 1990. A derivation of the coupling loss factors used in statistical energy analysis. Journal of Sound and Vibration 141 (2), 207 - 219.

Lyon, R., 2003. Statistical Energy Analysis of Dynamical Systems: Theory and Applications. Mit Press.

URL http://books . google . com/books? id=hHQRPwAACAAJ

Maksimov, D., Tanner, G., 2011. A New Hybrid Method to Predict the Distribution of Vibro-Acoustic Energy in Complex Built-Up Structures. Birkhauser Boston. 
McDonald, S. W., Kaufman, A. N., 1979. Spectrum and eigenfunctions for a Hamiltonian with stochastic trajectories. Phys. Rev. Lett. 42, 1189.

Mehta, M. L., 1991. Random Matrices, 2nd Edition. Academic Press, Boston.

Mello, P. A., Peveyra, P., Seligman, T. H., 1985. Information theory and statistical nuclear reactions. I. general theory and applications to few-channel problems. Ann. of Phys. 161, 254.

Mitchell, G. E., Richter, A., Weidenmuller, H. A., 2010. Rev. Mod. Phys. 82, 2845 .

Moglie, F., Primiani, V., april 2012. Numerical analysis of a new location for the working volume inside a reverberation chamber. Electromagnetic Compatibility, IEEE Transactions on 54 (2), $238-245$.

Orjubin, G., Richalot, E., Mengue, S., Picon, O., feb. 2006. Statistical model of an undermoded reverberation chamber. Electromagnetic Compatibility, IEEE Transactions on 48 (1), $248-251$.

Ott, E., 2002. Chaos in Dynamical Systems, 2nd Edition. Cambridge University Press, New York.

Pirkl, R., Ladbury, J., Remley, K., aug. 2011. The reverberation chamber's unstirred field: A validation of the image theory interpretation. In: Electromagnetic Compatibility (EMC), 2011 IEEE International Symposium on. pp. $670-675$.

Prange, R. E., 2005. Resurgence in quasi-classical scattering. J. Phys. A: Math. Gen. 38, 10703.

Primiani, V., Moglie, F., Paolella, V., aug. 2009. Numerical and experimental investigation of unstirred frequencies in reverberation chambers. In: Electromagnetic Compatibility, 2009. EMC 2009. IEEE International Symposium on. pp. $177-181$.

Primiani, V. M., Moglie, F., 2010. Numerical simulation of los and nlos conditions for an antenna inside a reverberation chamber. Journal of Electromagnetic Waves and Applications 24 (17-18), 2319-2331. 
Savin, D. V., Fyodorov, Y. V., Sommers, H.-J., 2001. Reducing nonideal to ideal coupling in random matrix description of chaotic scattering: Application to the time-delay problem. Phys. Rev. E 63, 035202(R).

Savin, D. V., Sommers, H.-J., 2004. Distribution of reflection eigenvalues in many-channel chaotic cavities with absorption. Phys. Rev. E 69, 035201(R).

Savin, D. V., Sommers, H.-J., Fyodorov, Y. V., 2005. Universal statistics of the local Green's function in wave chaotic systems with absorption. JETP Lett. 82, 544 .

Schäfer, R., Stöckmann, H.-J., Gorin, T., Seligman, T. H., Oct 2005. Experimental verification of fidelity decay: From perturbative to fermi golden rule regime. Phys. Rev. Lett. 95, 184102.

Schomerus, H., Jacquod, P., 2005. Quantum-to-classical correspondence in open chaotic systems. Journal of Physics A: Mathematical and General 38 (49), 10663.

Schwabl, F., Brewer, W., 2006. Statistical Mechanics. Advanced Texts in Physics. Springer.

URL http://books . google.com/books?id=7VnKAW284PgC

So, P., Anlage, S. M., Ott, E., Oerter, R. N., Apr 1995. Wave chaos experiments with and without time reversal symmetry: Gue and goe statistics. Phys. Rev. Lett. 74, 2662-2665.

Sridhar, S., Aug 1991. Experimental observation of scarred eigenfunctions of chaotic microwave cavities. Phys. Rev. Lett. 67, 785-788.

Stein, J., Stöckmann, H.-J., 1992. Experimental determination of billiard wave functions. Phys. Rev. Lett. 68, 2867.

Stöckmann, H.-J., Stein, J., 1990. "Quantum" chaos in billiards studied by microwave absorption. Phys. Rev. Lett. 64, 2215.

Tanner, G., Chappell, D., Hamdin, H. B., Giani, S., Seidel, C., Vogel, F., 2010. Acoustic energy distribution in multi-component structures - dynamical energy analysis versus numerically exact results. In: Proceedings of the ISMA 2010 and USD 2010. pp. $2435-2436$. 
Tanner, G., Sondergaard, N., 2007. Wave chaos in acoustics and elasticity. J. Phys. A 40 (50).

Türeci, H. E., Stone, A. D., Collier, B., Oct 2006. Self-consistent multimode lasing theory for complex or random lasing media. Phys. Rev. A 74, 043822. URL http://link.aps.org/doi/10.1103/PhysRevA.74.043822

Urbina, J. D., Richter, K., 2006. Statistical description of eigenfunctions in chaotic and weakly disordered systems beyond universality. Phys. Rev. Lett. 97, 214101.

Warne, L., Lee, K., Hudson, H., Johnson, W., Jorgenson, R., Stronach, S., may 2003. Statistical properties of linear antenna impedance in an electrically large cavity. Antennas and Propagation, IEEE Transactions on $51(5), 978-992$.

Weidenmüller, H. A., Mitchell, G. E., May 2009. Random matrices and chaos in nuclear physics: Nuclear structure. Rev. Mod. Phys. 81, 539-589.

Wigner, E., 1967. Random matrices in physics. SIAM Review 9 (1), 1-23.

Wigner, E. P., Dirac, P. A. M., 1950. On the statistical distribution of the widths and spacings of nuclear resonance levels. Proceedings of the Cambridge Philosophical Society 47, 790.

Wright, M., Weaver, R., 2010. New Directions in Linear Acoustics and Vibration: Quantum Chaos, Random Matrix Theory and Complexity. Cambridge University Press.

Yeh, J.-H., Antonsen, T. M., Ott, E., Anlage, S. M., 2012a. Fading statistics in communications - a random matrix approach. Acta Physica Polonica A $120, \mathrm{~A}-85$.

Yeh, J.-H., Antonsen, T. M., Ott, E., Anlage, S. M., 2012b. First-principles model of time-dependent variations in transmission through a fluctuating scattering environment. Phys. Rev. E(Rapid Communications) 85, 015202.

Yeh, J.-H., Hart, J. A., Bradshaw, E., Antonsen, T. M., Ott, E., Anlage, S. M., 2010a. Experimental examination of the effect of short ray trajectories in two-port wave-chaotic scattering systems. Phys. Rev. E 82, 041114. 
Yeh, J.-H., Hart, J. A., Bradshaw, E., Antonsen, T. M., Ott, E., Anlage, S. M., 2010b. Universal and nonuniversal properties of wave-chaotic scattering systems. Phys. Rev. E 81, 025201(R).

Zheng, X., 2005. Statistics of impedance and scattering matrices in microwave chaotic cavities: the random coupling model. Ph.D. thesis, University of Maryland, College Park.

Zheng, X., Antonsen, T. M., Ott, E., 2006a. Statistics of impedance and scattering matrices in chaotic microwave cavities: Single channel case. Electromagnetics 26,3 .

Zheng, X., Antonsen, T. M., Ott, E., 2006b. Statistics of impedance and scattering matrices of chaotic microwave cavities with multiple ports. Electromagnetics 26, 37 .

Zheng, X., Hemmady, S., Antonsen, T. M., Anlage, S. M., Ott, E., 2006c. Characterization of fluctuations of impedance and scattering matrices in wave chaotic scattering. Phys. Rev. E 73, 046208. 